Editorial

\title{
Acknowlegement to Reviewers of Advances in Environmental and Engineering Research in 2020
}

\author{
Advances in Environmental and Engineering Research Editorial Office
}

LIDSEN Publishing Inc., 2000 Auburn Drive, One Chagrin Highlands, Suite 200, Beachwood, OH, USA; E-Mail: aeer@lidsen.com

Adv Environ Eng Res

2021, volume 2, issue 1

doi:10.21926/aeer.2101001
Received: January 04, 2021

Accepted: January 04, 2021

Published: January 06, 2021

\begin{abstract}
The editors of Advances in Environmental and Engineering Research would like to express their sincere gratitude to the following reviewers for assessing manuscripts in 2020. We greatly appreciate the contribution of expert reviewers, which is crucial to the journal's editorial process. We aim to recognize reviewer contributions through several mechanisms, of which the annual publication of reviewer names is one. Reviewers receive a voucher entitling them to a discount on their next LIDSEN publication and can download a certificate of recognition directly from our submission system. Additionally, reviewers can sign up to the service Publons (https://publons.com) to receive recognition. Of course, in these initiatives we are careful not to compromise reviewer confidentiality. Many reviewers see their work as a voluntary and often unseen part of their role as researchers. We are grateful to the time reviewers donate to our journals and the contribution they make.
\end{abstract}

The editors of Advances in Environmental and Engineering Research would like to express their sincere gratitude to the following reviewers for assessing manuscripts in 2020.

We greatly appreciate the contribution of expert reviewers, which is crucial to the journal's editorial process. We aim to recognize reviewer contributions through several mechanisms, of which the annual publication of reviewer names is one. Reviewers receive a voucher entitling them to a discount on their next LIDSEN publication and can download a certificate of recognition directly from our submission system. Additionally, reviewers can sign up to the service Publons

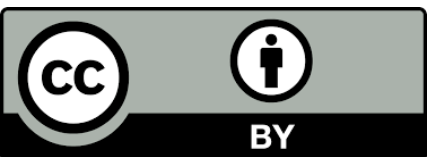

(C) 2021 by the author. This is an open access article distributed under the conditions of the Creative Commons by Attribution License, which permits unrestricted use, distribution, and reproduction in any medium or format, provided the original work is correctly cited. 
(https://publons.com) to receive recognition. Of course, in these initiatives we are careful not to compromise reviewer confidentiality. Many reviewers see their work as a voluntary and often unseen part of their role as researchers. We are grateful to the time reviewers donate to our journals and the contribution they make.

If you are interested in becoming a reviewer for Advances in Environmental and Engineering Research, see the link at the bottom of the webpage http://lidsen.com/joinus.

The following reviewed for Advances in Environmental and Engineering Research in 2020:

\begin{tabular}{|l|l|l|}
\hline Ali Hossein Mardi & Alicia García-Costa & Cheng Fang \\
\hline Christine Bingen & Corene Matyas & Dimitris G. Kaskaoutis \\
\hline James M. Tinjum & Johnsie R. Lang & Kathy Meney \\
\hline Linlu Mei & Martin C. Heller & Mohammad Boshir Ahmed \\
\hline Naomi Henderson & Neil I. Fox & Rebecca S. McWatters \\
\hline Roberto San Jose & Tomeu Rigo & Xiuhong Chen \\
\hline
\end{tabular}

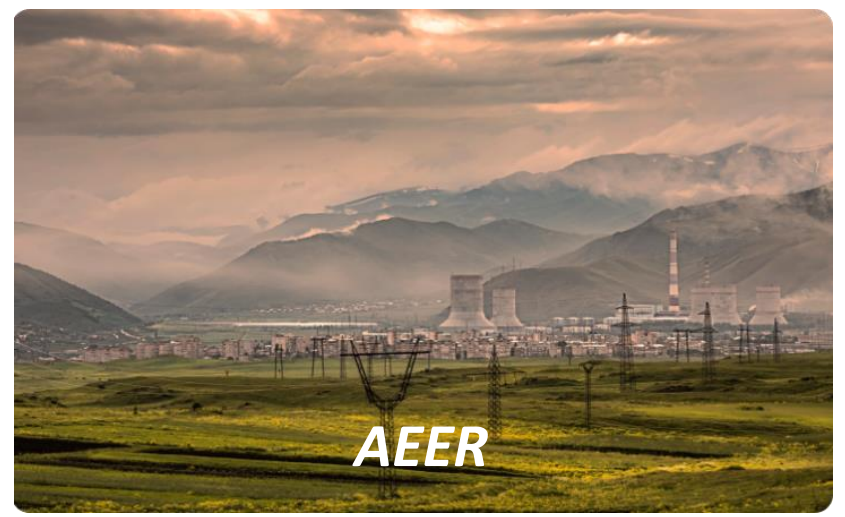

Enjoy $A E E R$ by:

1. Submitting a manuscript

2. Joining in volunteer reviewer bank

3. Joining Editorial Board

4. Guest editing a special issue

For more details, please visit:

http://www.lidsen.com/journals/aeer 